\title{
Para discutir la hegemonía epistémica evaluativa eurocéntrica: un enfoque exploratorio
}

\author{
Discussing Eurocentric evaluative epistemic hegemony: \\ an exploratory approach
}

Almerindo Janela Afonso'

\section{Resumen}

En el presente artículo pretendo, de una forma aún exploratoria, reunir algunos presupuestos de las teorías poscoloniales/descoloniales para elaborar un pensamiento crítico sobre la hegemonía de determinadas políticas, procesos e instrumentos de evaluación (o de medición educativa), que son claramente eurocéntricos (o euroamericanos) y que integran la actual agenda educativa global. Sin pretender contribuir para subsanar cualquier posible laguna pero reconociendo que, a pesar de las conocidas excepciones, el campo de las políticas de evaluación se encuentra relativamente saturado por abordajes repetitivos y previsibles, me resulta llamativa la capacidad hermenéutica de los presupuestos poscoloniales/descoloniales, cuya presencia en el campo del pensamiento crítico es cada vez más inevitable. Entre otros aspectos, quisiera llamar la atención sobre la urgencia en la construcción de relaciones y condiciones más justas y democráticas entre los diferentes países del sistema-mundial, especialmente en lo que se refiere a la producción del conocimiento, a través de la evaluación. En este sentido, si los países periféricos o semi-periféricos del sistema-mundial, en diferentes zonas del globo, adoptan de manera acrítica los indicadores definidos por el centro o no son capaces de resistir a la dominación cognitiva, o bien no cuestionan la integración subordinada en programas de evaluación comparativa internacional (como PISA y el AHELO), en cuanto instrumentos al servicio de una hegemonía epistémica eurocéntrica y euroamericana, la agenda educativa global continuará consolidándose únicamente como la expresión de los intereses del capitalismo, ampliando así, cada vez más, la colonialidad del conocimiento científico y evaluativo.

\section{Palabras clave}

Sistema mundial, eurocentrismo, presupuestos descoloniales, hegemonía evaluativa eurocéntrica.

\section{Abstract}

In this article I assemble, in an exploratory manner, some assumptions of postcolonial/decolonial theories so as to critically consider the hegemony of certain policies, processes and evaluation tools (or academic measurement) that make up the current global educational agenda. Without claiming to contribute to overcoming any possible gap, yet recognising that, in spite of known exceptions, the field of assessment policies is pretty much saturated with repetitive and predictable approaches, I feel called upon by the hermeneutic capacity of postcolonial/ decolonial assumptions, whose presence in the field of critical thinking is increasingly inescapable. One aspect to which I would like to draw attention is the urgency of building fairer and more democratic relations and conditions between the various nations of the world system, particularly with respect to the production of knowledge, and its evaluation. With this in mind, if the peripheral and semi-peripheral countries of the world system in different parts of the world uncritically adopt the indicators established by the centre, or are unable to resist cognitive domination, or agree to participate (even in a subordinate situation) in international comparative evaluation programmes (such as PISA and AHELO) as instruments in the service of Eurocentric and Euro-American epistemic hegemony, the global education agenda will continue to be consolidated solely as an expression of the interests of capitalism, thereby increasingly expanding the coloniality of evaluative and scientific knowledge.

\section{Key words}

World system, eurocentrism, decolonial assumptions, eurocentric evaluative hegemony. 


\section{Introducción}

Numerosos autores cuestionan de manera bastante contundente las formas de conocimiento hegemónico, o incluso llegan a defender un corte radical con la dependencia epistémica moderna, occidental y eurocéntrica. Como recuerda Walter Mignolo, «hoy, la descolonización ya no es un proyecto de liberación de las colonias, con el objetivo de dar lugar a la formación de estados nación independientes, sino más bien el proceso de descolonización epistémica y de socialización del conocimiento». (Mignolo, 2003:631).

Partiendo de este y de otros presupuestos de las teorías poscoloniales/descoloniales, pretendo elaborar un pensamiento crítico sobre la hegemonía de ciertos instrumentos y procesos de evaluación (o de medición educativa) que son claramente eurocéntricos (o euroamericanos) y se encuentran conectados con la agenda educativa global ${ }^{2}$. Se emplean diferentes designaciones en el campo de los estudios poscoloniales, aunque muchos de los presupuestos acaben siendo convergentes. Sin embargo, y en cualquiera de los casos, se trata de presupuestos cuyo conocimiento resulta insuficiente en determinadas áreas del campo educativo $^{3}$. Sin pretender contribuir para subsanar cualquier posible laguna pero reconociendo que, a pesar de las conocidas excepciones, el campo de las políticas de evaluación se encuentra relativamente saturado por abordajes repetitivos y previsibles, me resulta llamativa la capacidad hermenéutica de los presupuestos poscoloniales, cuya presencia en el campo del pensamiento crítico es cada vez más inevitable. Como afirma Grosfoguel, «hoy día resulta inexcusable para pensadores del Norte Global seguir produciendo teoría crítica sin diálogo, con sordera hacia las epistemologías del sur». (Grosfoguel, 2011:97).

Pretendo de esta forma en este texto, y de una manera aún exploratoria, dar seguimiento a un diálogo inter-epistémico que me parece muy sugerente y prometedor (también) para los abordajes al campo de las políticas educativas, en las que preferencialmente me sitúo. Más concretamente, realizaré una breve aproximación a componentes teórico-conceptuales poscoloniales, cuyos presupuestos políticos, culturales y epistemológicos pueden, desde mi punto de vista, aportar nuevas perspectivas al campo de la evaluación en educación, buscando una mayor comprensión sobre las políticas y las agendas actualmente hegemónicas en el contexto mundial.

\section{De la agenda nacional al giro hacia la agenda global: el papel de las organizacio- nes internacionales}

Efectivamente, si estas políticas empezaron teniendo un primer desarrollo (nacional) en países centrales, el impulso de la globalización y de la creciente internacionalización del capitalismo (donde se encuadra la acción de poderosas agencias e instancias internacionales y supranacionales) acabaron por reconfigurar estas agendas, ampliándolas también a países semi-periféricos y periféricos del sistema mundial.

Si consideramos las actuales políticas de evaluación (inicialmente impulsadas por los EE. UU. y por Inglaterra, y rápidamente adoptadas por diferentes países capitalistas occidentales), constataremos que estamos hoy en una fase sustancialmente diferente de aquella que Guy Neave (1988) inicialmente denominó

2 Ensayé inicialmente esta perspetiva en una conferencia de apertura que realicé en [desanonimizar]. el II Coloquio Cabo-Verdiano de Educação - Politicas e Práxis da Educação nas Perspetivas e em Contextos Pós-coloniais. Praia: Universidade de Cabo Verde, 24 de abril de 2015 y en una comunicación oral presentada en el congreso From decolonisation to postcolonialism: A global approach. Oporto: Facultad de Letras de la Universidade do Porto, 11-13 noviembre de 2015. La participación en estos eventos así como la actual publicación fueron apoyados por los fondos nacionales de la FCT- Fundação para a Ciência e Tecnologia - en el ámbito del proyecto PEst-OE/ CED/UI1661/2014, del Centro de Investigação em Educação da Universidade do Minho (Cied/UM).

3 En lo que dice respecto a la investigación portuguesa en educación (sobre todo en el dominio de la sociología de la educación y de las políticas educativas), se hace una referencia escasa a los trabajos de Ramón Grosfoguel, Walter Mignolo, Enrique Dussel, Alejandro Moreno, Aníbal Quijano, Stuart Hall, Edward Said, y muchos otros, a excepción de Maria Paula Menezes y de otros investigadores e investigadoras del Centro de Estudios Sociales de la Universidad de Coimbra, donde destaca Boaventura Sousa Santos como un autor de referencia obligatoria en este campo. 
como siendo la del contexto de emergencia del Estado-evaluador ${ }^{4}$. Efectivamente, la primera fase inicial del Estado-evaluador se circunscribe al contexto nacional, habiendo sido la evaluación un instrumento esencialmente de control y supervisión adoptado por diferentes gobiernos en el ámbito de la redefinición del papel del Estado -redefinición ésta que ha dado protagonismo a la evaluación como instrumento político, en articulación con otros instrumentos, dispositivos y modos de acción por entonces emergentes-. Éste es el caso, por ejemplo, de las formas emergentes de gobernanza que se fueron construyendo a través de rearticulaciones diversas entre pilares de regulación social (tradicionales) y nuevos actores colectivos, también sustentadas en redes de relaciones e interdependencias pluriescalares, con distribución descentralizada o desconcentrada de responsabilidades político-administrativas. Es también el caso de la diversificación y amplificación de formas de privatización; de la proliferación de discursos y modelos de accountability conservadores y tecnocráticos, así como de las agendas de la nueva gestión pública (new public management), entre otros.

En el sector público, considerado en sentido amplio, «la necesidad de desarrollar y perfeccionar herramientas de control, especialmente orientadas a la evaluación de los logros o resultados obtenidos, se asocia en la literatura a la nueva gestión pública, con la introducción de conceptos, prácticas y técnicas procedentes del sector privado». (García Sánchez, 2007:44). En el sector de la educación, más específicamente, la nueva gestión pública tuvo su expresión en «políticas como la autonomía de las escuelas, la profesionalización de los directores de las escuelas, la evaluación estandarizada y la rendición de cuentas de los profesores, y siendo ampliamente difundida por organizaciones internacionales como la OCDE» (Verger y Curran, 2014:253).

En lo que respecta a la evaluación en educación, sobre todo en el contexto de sus países miembros, la acción de la OCDE ha pasado a sentirse de forma más regular a partir del inicio de los años noventa (aunque haya sido ya esta misma organización la que haya conducido, muchos años antes, los famosos estudios de sistemas educativos nacionales). Así, si aún en la primera fase del Estado-evaluador varias decenas de estados, independientemente de la orientación político-ideológica de sus gobiernos, crearon, desarrollaron o reactualizaron sistemas nacionales de evaluación, empiezan ya a atisbarse las líneas de la transición hacia una nueva fase cuando, paralelamente a las pruebas y evaluaciones externas nacionales, estos estados van aumentando su participación en estudios internacionales basados en metodologías e indicadores esencialmente cuantitativos. Se convierten en práctica frecuente, por ejemplo, los informes periódicos Education at a Glance. La influencia de estos informes, tal como indica A. Teodoro, está bien presente en las políticas educativas de los estados miembros de la OCDE, desde la década de 1990. Se trata, según este autor, de «una influencia que se manifiesta no por un mandato explícito, sino por la necesidad de responder a una agenda global basada en la comparación y, sobre todo, en la competición de performance de los sistemas educativos». (Teodoro, 2012:28).

Por este motivo, organizaciones internacionales y supranacionales cada vez más pasan a incentivar dispositivos de evaluación comparativa internacional a gran escala, no solamente por la definición, perfeccionamiento y uso de indicadores cuantitativos, sino también por el uso de tests estandarizados. Los resultados de estos estudios comparativos han estado al servicio de diversos objetivos, entre los que se encuentran la construcción de una «narrativa legitimadora» para las políticas en educación adoptadas en el contexto interno (entre otros, Lingard, 2016:611).

4 Adopto la expresión Estado-evaluador para situar la problemática de la evaluación en su relación con el Estado y otras instancias de regulación y, en este sentido, es un uso más amplio que aquel que se hace por parte de Guy Neave, que acuñó la expresión esencialmente para análisis de la enseñanza superior. Para una perspectiva más amplia, especialmente en relación a la formación docente, ver también John Elliott (2002). 
En esta segunda fase del Estado-evaluador, además de informes como el Education at a Glance, el programa PISA (Programme for International Student Assessment) se convirtió en una referencia y ahora tiene «pretensiones normativas« (Beltrán Llavador, 2016: 130). Este programa ha sido una piedra angular en «la construcción de un espacio conmensurable de medición educativa a nivel global» (Rizvi y Lingard, 2010:133). Además de ésta, una otra contribución se está realizando a través del programa AHELO (Assessment of Higher Education Learning Outcomes), cuyo estudio de viabilidad ha sido recientemente concluido, también por la OCDE. Con el programa PISA se comparan países, mientras que con AHELO se compararán instituciones de enseñanza superior. Se trata, según R. Shahjahan, de un nuevo impulso para «desterritorializar la política de la enseñanza superior mediante la implementación de un régimen de tests transnacionales». También en este caso, al buscar «construir un espacio global de equivalencia para la enseñanza y el aprendizaje en la enseñanza superior» (Shahjahan, 2013:683), AHELO «perpetúa la colonialidad» del conocimiento (y su respectiva regulación), mediante una «retórica imperial» que sugiere que la educación superior global se encuentra en profunda crisis y que solamente se puede «salvan» incorporando realidades locales en modelos euroamericanos que, por su parte, al transformarse en universales, constituyen importantes instrumentos «para un nuevo orden mundial neoliberal vinculado al mercado de trabajo global». (Shahjahan, 2013:689).

Con el impacto que han tenido (y que es previsible que continúen a tener) los sucesivos estudios, recomendaciones y programas, no hay duda de que la OCDE es en la actualidad «el principal think tank mundial de la globalización hegemónica». (Teodoro, 2012:30).

Además del protagonismo de organizaciones como la OCDE, existen otras organizaciones económicas internacionales que se disputan la educación, incluso de una forma más agresiva y radical, obsesionadas con las agendas de la privatización y de la mercantilización de la educación a escala mundial. En este sentido, la acción de éstas últimas permite caracterizar una tercera fase (que he venido a denominar como de post-estado evaluador), en la que se hace cada vez más evidente la presión originada en instancias cuyo ejemplo paradigmático es la OMC - Organización Mundial del Comercio (Afonso, 2013).

No es casualidad que la OMC, después de considerar la educación como un servicio de elevado valor mercadeable y rentable, haya recibido contribuciones de algunos países donde surge la idea de extender los acuerdos do GATS a la evaluación y a los tests en educación -lo que, desde mi punto de vista, será inevitable que ocurra, dadas las potencialidades evidentes que los tests y pruebas estandarizadas tienen para ampliar los procesos de comercialización y de privatización y, en consecuencia, servir para el proceso de acumulación capitalista-.

Tal vez sea esta organización, más que ninguna otra, la que está radicalizando procesos que conducen de manera inevitable a la imposición (y no solo a la inducción) de una epistemología eurocéntrica en los países semi-periféricos y periféricos. Ya no se trata solamente del incentivo creciente a regímenes de franchising, sino también a la valorización de instrumentos estandarizados de evaluación, porque éstos son congruentes con la transferencia de modelos curriculares (originados en el Norte Global), los cuales, por lo tanto, se sobrepondrán o tenderán a ser ajenos a la autonomía relativa de los sistemas educativos nacionales y sus realidades históricas, políticas y culturales. Como si esto no bastase son ya muy visibles, en especial en países periféricos y semi-periféricos del sistema mundial, los efectos de la exportación y utilización de marcas creadas en países centrales. Estos efectos vienen asociados a la creación de monopolios privados de organizaciones de enseñanza (nacionales e internacionales), que resultan en la venta y fusión 
de escuelas, facultades y universidades, altamente lucrativos, y en muchos casos ya contados en las bolsas de valores (Bianchetti y Sguissardi, 2017).

\section{Colonialidad y comparativismo evaluador en países periféricos y semi-periféricos}

Como la ampliación de estas agendas se confunde con el movimiento creciente de internacionalización de las políticas de evaluación (y de la mayor frecuencia de uso de indicadores y otros instrumentos de medición), éstas tenderán a extenderse por todos los países, con independencia de su posición en el sistema mundial. En este sentido, el comparativismo evaluador se encuentra hoy presente no solamente en los países capitalistas centrales, sino también en los países semi-periféricos, en el contexto europeo (como es el caso de Portugal y España), o en los países semi-periféricos y periféricos de una parte del África subsahariana, de Asia y de América Latina.

Sin embargo, la densidad y el impacto de estas agendas es aún relativamente bajo, y muy diferenciado en lo que respecta especialmente a países de Asia y del África subsahariana. Por ejemplo, hasta el momento el informe PISA no ha salido completamente del ámbito de los países miembros (o asociados e invitados) de la OCDE (Sellan y Lingard, 2013). En su lugar, en lo que se refiere a la educación, tienen un peso mucho mayor los condicionantes y exigencias que ejercen organizaciones financieras tales como el Banco Mundial (BM) o el Fondo Monetario Internacional (FMI). Pero no son solamente éstos los aspectos que podrán explicar la (aún) inexpresiva participación en evaluaciones internacionales comparativas, aunque haya alguna excepción relativa al hecho de que algunos países del África subsahariana hayan participado en los estudios del Trends in International Mathematics and Science Study (por ejemplo, para el caso del TIMSS 2011, ver Bofah y Hannula, 2015). En el caso de África del Sur, después de muchas décadas de «aislamiento político y social, y de un legado de inequidad» (Howie, 2012:88), fue necesario dar prioridad a políticas contra la segregación racial y reorganizar un sistema educativo que estaba extremadamente fragmentado. Esto podrá explicar, al menos en parte, el hecho de que África del Sur solamente haya empezado a participar en estudios comparativos a partir del TIMSS 1995. Por otra parte, y al menos hasta 2004, este país se ha resistido a suscribir compromisos comerciales en el área de la educación, distanciándose de los acuerdos del GATS. En ese momento, además, se puede escribir: «Se trata de una posición significativa dado que este país exporta servicios educativos al resto del continente. Lo hace, en todo caso, en el ámbito de acuerdos bilaterales y en un marco de beneficio mutuo para los países implicados, y precisamente fuera del régimen de la política comercial». (Santos, 2004:37).

Más recientemente, con la creación del PISA-D (PISA para el desarrollo), al que algunos países semiperiféricos y periféricos, de África, América Latina y Asia ya se han unido, es de esperar que, de una forma más sistemática, se amplíen a estos y otros países de diferentes continentes los efectos globales de este programa de evaluación. Como indica el Director de Educación y Competencias de la OCDE, se trata de ofrecer «datos útiles para ayudar» a tomar decisiones relativas a los respectivos sistemas educativos y, por otro lado, «igualmente importante es que los países participantes en el PISA para el desarrollo se beneficiarán de intercambios entre pares con otros miembros de la comunidad global PISA». (Schleicher, 2016:1). Desde mi punto de vista, el deseo de contribuir para la creciente hegemonía del conocimiento científico y eurocéntrico relativo a la evaluación (y medición) en educación se encuentra aquí claramente presente en la idea, aparentemente benigna, de la afirmación de una «comunidad global PISA ${ }^{5} »$.

5 Una visión aún más optimista se desprende de la afirmación de M. Lockheed, cuando escribe: «El aumento del número de países en desarrollo que participan en las evaluaciones internacionales es indiscutible, gracias a la gran participación de los organismos donantes internacionales [...] una creciente necesidad de evidencias 
La cuestión, sin embargo, es mucho más compleja porque, tal como revela esta breve incursión en la literatura, las realidades nacionales están muy diferenciadas y, en muchos casos, poco o nada permeables a la adhesión a estas agentas. En el caso de muchos países del África subsahariana, por ejemplo, hay que tener en consideración que

«Las preocupaciones centrales en los contextos post-coloniales de educación son la identidady el idioma,
la formulación y la reformulación de politicas y regimenes post-coloniales, términos post-coloniales de
contestación discursiva, cambios en la naturaleza del Estado y nuevas teorías de derechos [...]. A
pesar de esto, muchos [autores] postulan agendas de investigación, aberturas conceptuales e indicadores
para estrategias politicas de cambio educativo que expresen elevados niveles de aspiración a un proyecto
post-colonial que aún no se ha realizado pero que, de algún modo, se encuentra inmanente en las ideas,
relaciones sociales y contestaciones que tienen lugar en la actualidad》. (Unterhalter, 2012:14).

En lo que se refiere, más específicamente, a la evaluación de los aprendizajes, hay países africanos que son más favorables a los exámenes, y otros que valorizan más la evaluación continua. Curiosamente, «la educación centrada en el alumno es una de las ideas educativas más difundidas en el África subsahariana». Como consecuencia de ello, las políticas educativas han intentado «disminuir el peso de los exámenes y aumentar la importancia de la evaluación continua como una forma de estimular pedagogías centradas en el alumno». (Chisholm y Leyendecker, 2012:54). En este contexto, habrá con certeza tensiones y contradicciones que es necesario entender y problematizar con mayor profundidad ${ }^{6}$.

De cualquier modo, en la perspectiva de algunos autores, esto es indicador de que el globo está siendo reconstruido como un espacio único de medición comparativa o como un espacio conmensurable del desempeño de los sistemas escolares, observándose lo que se puede denominar como «una forma de $p a$ noptismo global, con el ojo global funcionando en una capacidad reguladora a través y dentro de los estados nacionales». (Lingard et al., 2013:540).

Por este motivo, desde mi punto de vista la cuestión fundamental subyacente a este movimiento se enmarca claramente en el ámbito de una epistemología eurocéntrica o del Norte Global, y en la creencia en un único conocimiento universal. Como indica Grosfoguel, «El eurocentrismo se ha perdido en el camino del universalismo [...] que diluye todos los particulares en lo universal». Y, en la estela de Césaire, aquel autor añade aún que «el universalismo abstracto es lo que, a partir de un particularismo hegemónico, pretende constituirse como un proyecto global imperial para el mundo entero y que, al presentarse como desincorporado (“disembodied”), esconde el lugar epistémico de la enunciación». (Grosfoguel, 2012:100).

Este supuesto conocimiento universal es congruente con las lógicas de la internacionalización del capitalismo y con los (supuestos) proyectos de modernización, a los que sirven, directa o indirectamente,

\footnotetext{
de la "eficacia de la ayuda", ha impulsado la demanda de más países, particularmente países en desarrollo, para participar en evaluaciones de carácter internacional. [...] Este aumento se atribuye en parte al posicionamiento de los resultados del PISA en publicaciones dirigidas a economistas. [...] la participación en todos los tipos de evaluaciones internacionales ha beneficiado a los países en desarrollo tanto indirecta como directamente. Indirectamente, la participación ha influenciado las normas para el currículo y la práctica docente, llevando a una reforma curricular y dirigiendo la atención al desarrollo profesional de los maestros. Directamente, la participación ha fortalecido la capacidad de evaluación de sus organismos nacionales de evaluación, aportando formación relacionada con la evaluación, así como experiencia práctica sobre el proceso de elaboración y ejecución de evaluaciones a gran escala». (Lockheed, 2013:163).

6 Las tensiones y contradicciones no tienen lugar solamente en situaciones en que la colonialidad del poder se expresa en las relaciones Norte/Sur o en las relaciones centro/semiperiferia/periferia/centro, sino también en el interior del propio centro, de la propia semiperiferia o de la propia periferia. Las contribuciones que se vienen produciendo movilizando las teorías post-coloniales en torno a la problematización de las relaciones pedagógicas en la escuela pública de masas (predominantemente frecuentada por las clases populares) en un país semiperiférico como Brasil muestran, por ejemplo, que lo que se considera éxito o fracaso puede ser visto como expresión de la colonialidad del poder, siendo una de las principales funciones de la evaluación «naturalizar la diferencia colonial, que silenciosamente está presente en el cotidiano escolar, para justificar la selección y exclusión de sujetos, conocimientos y culturas». (Esteban 2010:51).
} 
las políticas de educación y de evaluación. Sobre este último aspecto, ciertas formas de evaluación comparativa (como el informe PISA) tienen, por lo menos, el apoyo tácito de las élites de países periféricos y semi-periféricos que continúan sin cuestionar muchos de los supuestos objetivos de modernización, seducidas ideológicamente por ímpetus de progreso (o de desarrollo) solamente susceptibles de ser realizados en conexión con agendas educativas de países capitalistas centrales o de elevado desarrollo (a pesar de que estas agendas parezcan neutras o benévolas, al ser transmitidas por organizaciones internacionales de prestigio tales como la OCDE o el Banco Mundial) 7 .

La creencia de que no hay alternativas más allá del capitalismo liberal es congruente no solo con el a-historicismo vigente en el occidente europeizado y americanizado, sino que también es congruente con la adopción de orientaciones políticas, culturales y económicas que parecen presuponer un único patrón de referencia -el de los países capitalistas centrales y/o países más desarrollados y competitivos, y supuestamente más cultos y más educados-. Dicho de otra forma, estos discursos sugieren que los mismos niveles de educación y conocimiento científico, y las mismas oportunidades de inserción ventajosa en una economía global de elevado carácter competitivo resultan accesibles a todos los países, siendo para esto necesario que las evaluaciones comparativas nacionales o los tests estandarizados internacionales indiquen (objetivamente) los déficits a superar y los caminos a seguir.

Pero las desigualdades entre países no se superan con evaluaciones comparativas basadas en tests estandarizados. A pesar de que existan fuertes tendencias para que estos instrumentos encuentren su justificación a la luz de la evolución de las metodologías y técnicas de evaluación (ellas propias producidas por el conocimiento científico hegemónico), pienso que es pertinente apelar aquí (recontextualizándola) a la observación de Immanuel Wallerstein cuando apuntaba que a los países situados en posiciones periféricas y semiperiféricas en la jerarquía del sistema mundial «se les incita a superar el hándicap de su bajo status» asumiendo valores supuestamente universales. Y añade: «Haciéndose competitivos en el mercado, los individuos y los grupos pueden obtener lo que otros ya tienen, y así alcanzarán algún día la igualdad; mientras tanto, la desigualdad resulta inevitable». (Wallerstein, 2004:263) ${ }^{8}$.

Si el ansia ilimitada por el lucro y la acumulación forma parte del ethos del sistema capitalista, es importante cuestionarnos por qué razón los países centrales (o las organizaciones internacionales más poderosas en la construcción de la agenda global) están interesadas en la subida de los niveles educativos de los países periféricos y semi-periféricos. Una respuesta posible, entre otras muchas, puede remitir para el hecho de que sea deseable el desarrollo de esto países, siempre que este desarrollo tenga lugar como condición para la continua expansión capitalista, especialmente en la preparación e integración de nuevos consumidores, lo antes posible, en el mercado global de la enseñanza (ahora ya asumido y sin ningún escrúpulo, a modo de bien transaccionable y de elevado valor mercantil) así como también en el mercado de trabajo global.

Sabiendo que hay vínculos frecuentemente convergentes (aunque no siempre claros) entre los intereses del capitalismo y los objetivos de muchas organizaciones internacionales, la acción de éstas últimas no puede pensarse aceptando de manera acrítica los discursos que ellas mismas elaboran y las misiones que

\footnotetext{
Para un interesante abordaje sobre el papel de las élites en un país africano, ver por ejemplo Sumich (2008).

8 Aunque el lugar de los diferentes países en el sistema mundial pueda modificarse (y se modifique), la tendencia es que, una vez definida la división internacional del trabajo, esa situación se reproduzca: «Esta posición puede ser sintetizada en la idea de que existe efectivamente una división internacional del trabajo, que ha sido establecida por un recorrido histórico de consolidación del capitalismo como un sistema global a lo largo de aproximadamente doscientos años; más aún, que esta división internacional del trabajo se reproduce indefinidamente, a menos que se alteren aspectos estructurales». (Almeida Filho, 2011:37).
} 
anuncian. En este sentido, podemos y debemos interrogarnos en relación al exacerbado (y aparentemente benévolo) protagonismo de organismos internacionales, tales como la OCDE y el Banco Mundial que, de forma creciente, se aceptan como incuestionables y supuestamente imprescindibles en la estructuración de políticas educativas nacionales y en la formulación de muchas otras decisiones. También por este motivo, pensar las políticas de evaluación en educación de una forma crítica puede encontrar, en las perspectivas poscoloniales, contribuciones originales que justifiquen atravesar las habituales fronteras de la investigación y reflexión en este campo.

Desde mi punto de vista, es precisamente esta relación de dominación cognitiva la que continúa sustentando muchas de las opciones de la política educativa y muchos de los modelos de evaluación adoptados en diferentes sistemas educativos, sobre todo los de países que tienen una posición periférica o semiperiférica en el contexto mundial. En el fondo, lo que está en cuestión es saber si es posible (y deseable) pensar en la evaluación poniendo de lado la referencia al conocimiento científico moderno y las teorías dominantes, es decir, fuera de cualquier dependencia epistémica occidental u occidentalizada.

Desde mi punto de vista sí que es posible y deseable, a pesar de que la evaluación en educación y, sobre todo aquella que se presenta como científica y técnicamente avanzada (como es el caso de los tests estandarizados) continúe siendo un instrumento de la supremacía epistémica evaluativa eurocéntrica, que se mantendrá seguramente de esta forma durante más tiempo como uno de los instrumentos actuales de la globalización capitalista o incluso de neocolonialismo en un contexto de post-colonización, hasta el momento en que se puedan fortalecer agendas alternativas. Además, más allá del currículo, pocas áreas serán tan universalizables y homogeneizables como la evaluación. Y es esta posibilidad de universalización y de homogeneización la que la ha difundido como uno de los virus del neoliberalismo en educación.

De nuevo, una vuelta al cientifismo neopositivista le queda bien a las lógicas capitalistas y las llamadas políticas basadas en evidencias (Afonso, 2015). No es por casualidad que estas políticas indujeron e inducen al uso de indicadores cuantificables y medibles, que han sido una de las estrategias privilegiadas por las organizaciones internacionales y supranacionales. Es más, si vamos atrás en el tiempo, tal como llama la atención uno de los autores que escribe a partir de los postulados de las teorías poscoloniales, las decisiones basadas en la evidencia tienen sus raíces en los procesos (iluministas) de burocratización, estandarización y normalización que tuvieron lugar de forma concomitante con el comercio de esclavos y el sistema de plantaciones, los cuales constituyeron experiencias de disciplinación e ingeniería social, de producción en serie, de sistematización de la vida humana y de institucionalización de la estandarización de procesos (Shahjahan, 2011). En el contexto actual y de una forma más genérica, «la objetivación numérica de fenómenos sociales» sirve también para «despolitizar las cuestiones políticas al naturalizar determinadas interpretaciones de la realidad en detrimento de visiones alternativas». (Erkkila y Piironen, 2014:344).

Por este motivo, la cuestión que se levanta una vez más es la de saber quién tiene el poder de definir los indicadores que cuentan para la comparación entre países; qué países participan en la definición de estos indicadores; y si no deberíamos ser capaces de pensar indicadores alternativos a los indicadores actualmente hegemónicos. Pero hay ya una importante reflexión que va más lejos, cuestionando «si no deberíamos comenzar a pensar no tanto, o no solo, en la construcción de "indicadores alternativos", sino más bien en una alternativa a los indicadores». (Beltrán Llavador, 2012:49). En cualquier caso, sabemos que no todos los países participan (o tienen el poder de interferir) en la definición y construcción de 
indicadores, pero cuando las decisiones políticas y administrativas están basadas en cuantificaciones numéricas, sus premisas no podrían dejar de estar «visibles, accesibles y abiertas a la deliberación pública» (Erkkila y Piironen, 2014:346).

Tal como en el caso de los tests estandarizados, los indicadores pueden ser vehículos de un «nuevo imperialismo que refleja los intereses de las naciones occidentales y, más ampliamente, del capitalismo global». (Nguyen et al., 2009:110). Y si los países periféricos o semi-periféricos del sistema-mundial, en diferentes zonas del globo, adoptan de manera acrítica los indicadores definidos por el centro, o bien no son capaces de resistir a la dominación cognitiva, o no cuestionan la integración subordinada en programas de evaluación comparativa internacional (tales como PISA y AHELO), en cuanto instrumentos al servicio de una hegemonía epistémica eurocéntrica y euroamericana, la agenda educativa global continuará consolidándose como la expresión de los intereses del capitalismo, ampliando así, cada vez más, la colonialidad del conocimiento científico y evaluativo.

\section{Referencias bibliográficas}

Afonso, Almerindo J. (2013): "Mudanças no Estado-avaliador: comparativismo internacional e teoria da modernização revisitada”. Revista Brasileira de Educação, 18 (53), 267-284.

Afonso, Almerindo J. (2015): "Recuo ao cientificismo, paradoxos da transparência e corrupção em educação”. Educação e Pesquisa, 41 (n especial), 1313-1326.

Almeida Filho, Niemeyer (2011): "Os países em desenvolvimento estão se tornando desenvolvidos? Crítica e Sociedade. Revista de cultura politica, 1 (2), 35-50.

Beltrán Llavador, José (2012): “¿Indicadores alternativos o alternativa a los indicadores? Alguns reflexiones sobre política educativa” en António Teodoro y Edineide Jezine (eds.): Organizações internacionais e modos de regulação das políticas de educação. Indicadores e comparações internacionais. Brasília: Liber Livro.

Beltrán Llavador, José (2016): "Medida y valor de la escuela. Desde la rendición de cuentas hasta la rendición de sentido". Profesorado - Revista de curriculum y formación del profesorado, 20 (3), 119-143.

Bianchetti, Lucídio y Sguissardi, Valdemar (2017). Da universidade à commoditycidade. Campinas: Mercado das Letras.

Bofah, Emmanuel Adu-tutu y Hannula, Markku S. (2015): "TIMSS data in an African comparative perspective: Investigating the factors influencing achievement in mathematics and their psychometric properties". Large-Scale Assessments Education, 3 (4), 1-36.

Chisholm, Linda y Leyendecker, Ramon (2012): “A reforma do currículo na África subsariana: quando o local encontra o global” en Robert Cowen et al., (eds.): Educação Comparada-panorama internacional e perspectivas. Brasília: CAPES/UNESCO.

Elliott, John (2002): “The paradox of educational reform in the evaluatory State: Implications fot teacher education”. Prospects, 32 (3), 273-287.

Erkkila, Tero; Piironen, Ossi (2014): “(De)politicizing good governance: the World Bank Institute, the OECD and the politcs of governance indicators". Innovation: The European Journal of Social Science Research, 27 (4), 344-360. 
Esteban, M. Teresa (2010): "Diferença, aprendizagens e avaliação: perspectiva pós-colonial e escolarização” en M. Teresa Esteban y Almerindo J. Afonso (eds): Olhares e Interfces. Reflexões críticas sobre a avaliação. São Paulo: Cortez.

García Sánchez, Isabel María (2007): "La nueva gestión pública: evolución y tendências”. Presupuesto y Gasto Público, 47, 37-64.

Grosfoguel, Ramón (2011): "La descolonización del conocimiento: diálogo crítico entre la visión descolonial de Frantz Fanon y la sociología descolonial de Boaventura de Sousa Santos” en Formas-Otras: Saber, nombrar, narrar, hacer. Barcelona: CIDOB Edicions.

Grosfoguel, Ramón (2012): “Decolonizing Western Uni-versalisms: Decolonial Pluri-versalism from Aimé Césaire to the Zapatistas". Transmodernity. Journal of Peripheral Cultural Production of the Luso-Hispanic World, 1 (3), 88-104.

Howie, Sara (2012): "High-stakes testing in South Africa: friend or foe?" Assessment in Education: Principles, Policy \& Practice, 19 (1), 81-98.

Lingard, Bob (2016): "PISA: fundamentações para participar e acolhimento político". Educação \& Sociedade, 37 (136), 609-627.

Lingard, Bob, Martino, Wayne, Rezai-Rashti, Goli (2013): “Testing regimes, accountabilities and education policy: commensurate global and national developments". Journal of Education Policy, 28 (5), 539-556.

Lockheed, Marlaine (2013): "Causes and consequences of international assessments in developing countries” en Heinz-Dieter Meyer y Aaron Benavot (eds.): PIS A, Power, and Policy the emergence of global educational governance. Oxford: Symposium Books.

Mignolo, Walter (2003): “Os esplendores e as misérias da «ciência»: colonialidade, geopolítica do conhecimento e pluri-versalidade epistémica” en Boaventura S. Santos (comp.): Conbecimento prudente para uma vida Decente: Um discurso sobre as ciências revisitado. Porto: Afrontamento.

Neave Guy (1988): "On the cultivation of quality, efficiency and enterprise: an overview of recent trends in higher education in Western Europe, 1986-1988”. European Journal of Education, 23 (1/2), 7-23.

Nguyen, Phuong-Mai, Elliott, Julian, Terlouw, Cees, Pilot, Albert (2009): “Neocolonialism in education: Cooperative learning in Asian context". Comparative Education, 45(1),109-130.

Rizvi, Fazal y Lingard, Bob (2010). Globalizing education policy. New York: Routledge.

Santos, Boaventura S. (2004). A universidade no século XXI. Para uma reforma democrática e emancipatória da universidade. São Paulo: Cortez.

Schleicher, Andreas (2016). "Prólogo". En OCDE (2016). Pisa para el Desarrollo. https://www.oecd.org/ pisa/pisa-for-development/PISA-D_brochure_2016_ESP.pdf

Sellan, Sam y Lingard, Bob (2013): “The OECD and global governance in Education". Journal of Eduction Policy, 28 (5), 710-725.

Shahjahan, Riyad (2011): "Descolonizing the evidence-based education and policy movement: revealing the colonial vestiges in educational policy, research, and neoliberal reform". Journal of Education Policy, $26(2), 181-206$. 
Shahjahan, Riyad (2013): "Coloniality and a global testing regime in higher education: unpacking the OECD's AHELO initiative”. Journal of Education Policy, 28 (5), 676-694.

Sumich, Jason (2008). "Construindo uma nação: ideologias de modernidade da elite moçambicana”. Análise Social, 43 (2),319-345.

Teodoro, António (2012): “Os novos modos de regulação transnacional das políticas de educação: a regulação pelos resultados e o papel das comparações internacionais” en António Teodoro y Edineide Jezine (eds.): Organiz̧ações Internacionais e Modos de Regulação das Políticas de Educação. Indicadores e comparações internacionais. Brasília: Liber Livro.

Unterhalter, Elaine (2012): "Introdução editorial da secção" en Robert Cowen et al., (eds.): Educaşão Comparada-panorama internacional e perspectivas. Brasília: CAPES/UNESCO.

Verger, Antoni y Curran, Marta (2014): "New public management as a global education policy: its adoption and re-contextualization in a Southern European setting”. Critical Studies in Education, 55 (3), 353-271.

Wallerstein, Immanuel (2004). Capitalismo histórico y movimientos antisistémicos. Un análisis de sistemas-mundo. Madrid: Akal.

\section{Nota biográfica}

Almerindo Janela Afonso es sociólogo y profesor de la Universidade do Minho, Portugal, donde imparte clase desde 1985. Ha investigado y ejercido docencia en las áreas de sociología de la educación y políticas educativas, entre otras. Ha ejercido varios cargos de gestión científico-pedagógica y es director del Departamento de Ciencias Sociales de la Educación y miembro del comité directivo del Doctorado en Ciencias de la Educación. Ha recibido el premio «Rui Gracio», otorgado por la Sociedad Portuguesa de Ciencias de la Educación y la Fundación Calouste Gulbenkian. Entre otros trabajos, es autor de Avaliação Educacional: Regulação e Emancipação. São Paulo: Cortez Editora, 2014; coorganizador de Olhares e Interfaces. Reflexões Críticas sobre a Avaliação. São Paulo: Cortez, 2013; Fragmentos de Escrita Pública. Porto: Profedições, 2013. 